\title{
Periodic Solutions of Ordinary Differential Equations and the Rotation of Associated Vector Fields (*).
}

\author{
Hexmut KNolle (Bochum, German Federal Republic)
}

Sunto. - I criteri di esistenza di soluzioni periodiche dell'equazione $\dot{x}=f(x, t), x \in R^{n}$ richiedono spesso le stesse condizioni per tutte le componenti di $f$; per esempio se $f(x, t)=A x+g(x, t)$ sia $\lim _{|x| \rightarrow \infty} g(x, t) /|x|=0 . t$. nella sezione III di questo lavoro impone questa condizione per $p<n$ componenti, mentre le altre $n-p$ soddisfano ad una condizione del tipo

$$
x f(x, t)<0 \quad \text { per }|x| \geqslant R .
$$

Anche nella Sezione II l'A. dà un criterio di esistenza di soluzioni periodiche facendo per aloune componenti delle ipotesi come la (*) ma più deboli, e con opportune ipotesi per le altre componenti. I procedimenti dimostrativi, di natura topologioa e funzionale, si fondano su un teorema di Krasnoselski che stabilisce una relazione tra la rotazione di un campo di vettori in $R^{n}$ e la rotazione della proiezione del campo in un sottospazio lineare di $R^{n}$.

\section{1. - Introduction.}

To many ordinary differential equations $\dot{x}=f(x, t)$ where the right side is periodie in $t$, we can associate a continuous vector field in the phase space or a completely continuous vector field in an appropriate function space. There are equivalence theorems, by which the periodic solutions of the differential equation correspond to the zcros of the associated vector field (see for example REISsIg [4]). A sufficient condition for a vector field to have zeros in some region is a nonzero rotation on the boundary of the region.

When estimating the rotation of a vector field, it is sometimes useful to split the field into two components. For in certain cases the rotation of the original field has the same parity as the rotation of one of its components. This is, very roughly, the meaning of a theorem by KrasNoseLsKI ([1], p. 99). We'll formulate this theorem, which is a generalization of Borsuk's theorem about antipodes. In the following let $U$ be a linear subspace of $R^{n}$ and $P$ the projection operator associated to it. Define the operator $*$ by

$$
x^{*}=2 P x-x
$$

(*) Entrata in Redazione il 20 dicembre 1973.

16 - Annali di Matematica 
With these notations we state the

Theorem. - Suppose there is given a vector field $\Phi(x)$ on a $(n-1)$ sphere $S \subset R^{n}$ whose center is in $U$. If the conditions

1) $\Phi \neq 0$ on $S$;

2) $P \Phi \neq 0$ on $S \cap U$;

(D)

3) $\Phi\left(x^{*}\right)$ and $\Phi(x)^{*}$ have nowhere on $S$ opposite direction

are satisfied, then the rotation of $\Phi$ on $S$ has the same parity as the rotation of $P \Phi$ on $B \cap U$.

The third condition shall be mentioned as condition (D) (direction condition).

We'll apply this theorem to nonlinear differential equations whose right sides split into two components of different behaviour. In many former proofs of existence of periodic solutions, all scalar components of the right side have the same property, for example $f(x, t)=A x+g(x, t)$ with

$$
\lim _{x \rightarrow \infty} \frac{g(x, t)}{|x|}=0 \text { and an appropriate matrix } A
$$

We meet this condition again in section III, but it is imposed only on $p<n$ scalar components, whilest the other $n-p$ components satisfy an essentially different condition.

\section{2. - Operating in phase space.}

For convenience we will give condition (D) a more practicable form. If $U$ is the subspace defined by $x_{p+1}=\ldots=x_{n}=0(0<p<n)$, let $u$ be the vector $\left(x_{1}, \ldots, x_{p}\right)$ and $v$ the vector $\left(x_{p+1}, \ldots, x_{n}\right)$. We may write

$$
\Phi(x)=\left\{\begin{array}{l}
f(u, v) \\
g(u, v)
\end{array}\right\}
$$

where $f$ has $p$ and $g$ has $n-p$ components. Then condition (D) can be formulated as follows:

For every $\lambda>0$ and every $(u, v) \in S$

$$
\begin{aligned}
f(u,-v) & \neq-\lambda f(u, v) \\
\text { or } \quad g(u,-v) & \neq \lambda g(u, v) .
\end{aligned}
$$


HeLmut KnolLe: Periodio solutions of ordinary differential equations, ete. $\quad 239$

Now let us consider the system of differential equations

$$
\begin{aligned}
& \dot{x}=f(x, y, t) \\
& \dot{y}=g(x, y, t)
\end{aligned}
$$

where $x$ is a $p$-vector $(0<p<n)$ and $y$ a $(n-p)$-vector, $f$ and $g$ are continuous in their arguments, periodic in $t$ with period $\omega$ and satisfy a Lipschitz or a similar condition that guarantees the uniqueness for the initial value problem. Write $z=(x, y)$ and let $Z\left(t, z, t_{0}\right)$ be the unique solution of $(1)$ satisfying $Z\left(t_{0}, z, t_{0}\right)=z$. If on some sphere $S \subset R^{n}$ the rotation of the field

$$
\Phi(z)=(f(x, y, 0), g(x, y, 0))
$$

is nonzero, and if

$$
Z\left(t, z, t_{0}\right) \neq z \quad \text { for all } z \in S \text { and } t_{0}<t \leqslant t_{0}+\omega
$$

then the system (1) has at least one $\omega$-periodic solution (v. Purss [3]).

We will prove the following

Proposition 1. - If there exist numbers $r>0$ and $\alpha>0$ such that

a) $x f(x, y, t)>0$ for $|y| \leqslant \alpha|x|,|x|>r, 0 \leqslant t \leqslant \omega$

b) $y g(x, y, t)>0 \quad(<0)$ for $|y| \geqslant \alpha|x|,|y|>r, 0 \leqslant t \leqslant \omega$

then the system (1) has at least one $\omega$-periodic solution.

Proof. - We put $R=\left(1+\alpha+\alpha^{-1}\right) r$ and show at first, that condition (3) is satisfied on the sphere $S$, defined by $|z|^{2}=|x|^{2}+|y|^{2}=R^{2}$. Denote by $A$ the sector where $|y|<\alpha|x|$ and by $B$ the sector where $|y|>\alpha|x|$. If $z_{0}=\left(x_{0}, y_{0}\right) \in S \cap \bar{A}$, then $\left|x_{0}\right|^{2}=R^{2}-\left|y_{0}\right|^{2} \geqslant R^{2}-\alpha^{2}\left|x_{0}\right|^{2}$ and $\left(1+\alpha^{2}\right)\left|x_{0}\right|^{2} \geqslant R^{2}>\left(1+\alpha^{2}+\alpha^{-2}\right) r^{2}>\alpha^{-2}\left(1+\alpha^{2}\right) r^{2}$. If $z_{0} \in \mathbb{S} \cap \bar{B}$, then $\left|y_{0}\right|^{2} \geqslant R^{2}-\alpha^{-2}\left|y_{0}\right|^{2}$ and

$$
\left(1+\alpha^{-2}\right)\left|y_{0}\right|^{2} \geqslant R^{2}>\left(1+\alpha^{2}+\alpha^{-2}\right) r^{2}>\alpha^{2}\left(1+\alpha^{-2}\right) r^{2} .
$$

We have shown

$$
\begin{array}{lll}
z_{0} \in S \cap \bar{A} & \text { implies } & \left|x_{0}\right|>\max \left(r, \alpha^{-1} r\right) \\
z_{0} \in S \cap \bar{B} & \text { implies } & \left|y_{0}\right|>\max (r, \alpha r) .
\end{array}
$$

When the trajectory $Z\left(t, z_{0}, t_{0}\right)$ returns to $z_{0}$, then for at least one $t_{1}>t_{0}$

$$
d / d t\left(x^{2}\right)_{t_{3}}=0 \quad \text { and } \quad\left|x\left(t_{1}\right)\right| \geqslant\left|x_{0}\right|
$$


and for at least one $t_{2}>t_{0}$

$$
d / d t\left(y^{2}\right)_{t_{2}}=0 \text { and } \quad\left|y\left(t_{2}\right)\right| \geqslant\left|y_{0}\right| .
$$

Now if $z_{0} \in S \cap \bar{A}$, then (4) and (5) imply $x f\left(x, y, t_{1}\right)=0$ and $\left|x\left(t_{1}\right)\right|>r$, hence by a) $Z\left(t_{1}, z_{0}, t_{0}\right) \notin \bar{A}$. If $z_{0} \in S \cap B$, then (4) and (6) imply $y g\left(x, y, t_{2}\right)=0$ and $\left|y\left(t_{2}\right)\right|>r$, hence by $b) Z\left(t_{2}, z_{0}, t_{0}\right) \notin \bar{B}$. Therefore a trajectory returning to its starting point $z_{0} \in S$, if it exists, must contain points of both sets $A$ and $B$.

Now let $Z\left(t, z_{0}, t_{0}\right)$ with $z_{0} \in \bar{A} \cap S$ enter the open set $B$ at the time $t_{1}$ and leave it at the time $t_{2}>t_{1}$ (when it rests in $B$, it is impossible to retun to $z_{0}$ ). If the $>$ in condition $b$ ) is assumed, we have

$$
r<\left|x\left(t_{0}\right)\right|<\left|x\left(t_{1}\right)\right|=\alpha^{-1}\left|y\left(t_{1}\right)\right|<\alpha^{-1}\left|y\left(t_{2}\right)\right|=\left|x\left(t_{2}\right)\right| .
$$

So $|x(t)|$ increases still for $t>t_{2}$ until the trajectory enters $B$ again, and so on. Therefore the trajectory cannot return to its starting point. The same argument can be applied when $z_{0} \in B \cap S$. If the $<$ in condition $b$ ) is assumed, we remark that by condition $a$ ) and the continuity of $f, x^{2}$ and $|x|$ still increase in some time intervall after $t_{1}$. So for some $h, 0<h<t_{2}-t_{1}$, we have

$$
\left|y\left(t_{1}\right)\right|=\alpha\left|x\left(t_{1}\right)\right|<\alpha\left|x\left(t_{1}+h\right)\right|<\left|y\left(t_{1}+h\right)\right|
$$

and by Rolle's theorem it follows, that for some $\tau, t_{1}<\tau<t_{1}+h$, we have $d / d t\left(y^{2}\right)=$ $=y g(x, y, \tau)>0$. But by $(4)$

$$
r<\alpha\left|x\left(t_{0}\right)\right|<\alpha\left|x\left(t_{1}\right)\right|<\alpha|x(\tau)| \leqslant|y(\tau)|
$$

in contradiction to $b)$. Therefore the assumption, that $Z\left(t, z_{0}, t_{0}\right)$ enters $B$, was false. Assume still the $<$ in condition $b$ ) and let a trajectory start in $z_{0} \in B \cap S$, enter $\bar{A}$, leave it at the time $t_{1}$ and return to $z_{0}$. Then

$$
\max (r, \alpha r)<\left|y\left(t_{0}\right)\right|<\left|y\left(t_{1}\right)\right|=\alpha\left|x\left(t_{1}\right)\right|
$$

hence $\left|x\left(t_{1}\right)\right|>r$. We have again the situation, that a trajectory enters $B$ when $d / d t\left(x^{2}\right)$ is still positive, and this implies the same contradiction as before. Thus we have shown, that a trajectory returning to its starting point on $S$ cannot contain points of $A$ and $B$. Therefor condition (3) is fulfilled on 8 .

It rests to prove that the rotation of the vector field (2) on $S$ is nonzero. We observe that, according to $a$ ) and $b), \Phi(z)$ does not vanish on $S$. To apply the generalized Borsuk theorem we must show that condition $\left(D^{\prime}\right)$ is satisfied on $S$.

Let $z \in \bar{A} \cap S$ and $f(x,-y, 0)=-\lambda f(x, y, 0)$ for $\lambda>0$. Multiplying by $x$ we see that $x f(x, y, 0)$ and $x f(x,-y, 0)$ have opposite sign, in contradiction to condition $a)$. 
HeLmut KNoLLE: Periodic solutions of ordinary differential equations, eto. 241

So the first inequality of $D^{\prime}$ is satisfied. In the case $z \in B \cap S$, the second inequality is satisfied. This becomes obvious, when we multiply $g(x,-y, 0)=\lambda g(x, y, 0)$ by $-y$, getting a contradiction to condition $b)$ which says that $y g(x, y, 0)$ and $-y g(x,-y, 0)$ have the same sign.

The subspace defined by $y=0$ is called $U$. The sphere $S \cap U$ lies entirely in $A$, so we have on it $x f(x, 0,0)>0$. Therefore the rotation of $f(x, 0,0)$ on $S \cap U$ is defined and equal to 1 by the Poincaré-Bohl theorem. Now the generalized Borsuk theorem implies that the rotation of the field $\Phi$ on $S$ is an odd number and especially nonzero. This complets the proof.

EXAMPLE. - We consider a system of two scalar equations

$$
\begin{aligned}
& \dot{x}=x^{n}+\sum_{i+\dot{k}=n}^{0 \leqslant k<n} a_{i k}(t) x^{i} y^{\bar{k}}+P_{n-1}(x, y, t) \\
& \dot{y}= \pm y^{m}+\sum_{i+k=m}^{0 \leqslant i<m} b_{i k}(t) x^{i} y^{i k}+P_{m-1}(x, y, t)
\end{aligned}
$$

where $n$ and $m$ are odd, $P_{n-1}$ and $P_{n-1}$ are polynomials of degree not greater than $n-1$ resp. $m-1$ with coefficients $\omega$-periodie in $t$ and the $a_{i k}$ and $b_{i k}$ are $\omega$-periodic in $t$ and such that for some $\alpha>0$ and $0 \leqslant t \leqslant \omega$ we have

$$
\begin{array}{ll}
\sum\left|a_{i k}(t)\right| \alpha^{k} \leqslant 1-\delta, & \delta>0 \\
\sum\left|b_{i k}(t)\right| \alpha^{-i} \leqslant 1-\varepsilon, & \varepsilon>0
\end{array}
$$

with $i$ and $k$ running over the same range as above. Then in the sector $\alpha|x| \geqslant|y|$ we have

$$
\left|x^{n}\right|+a_{i k} x^{i} y^{k} \geqslant\left|x^{n}\right|-\left|x^{n}\right| \sum\left|a_{i k}\right| \alpha^{k} \geqslant \delta\left|x^{n}\right|
$$

and the last term increases stronger than $P_{n-1}$. For $\alpha|x| \leqslant|y|$ we have $\left|y^{m}\right|+$ $+\sum b_{i k} x^{i} y^{k} \geq \varepsilon\left|y^{m}\right|$. Since $n$ and $m$ are odd, conditions $a$ ) and $b$ ) of proposition 1 . are satisfied for $r$ great enough.

\section{3. - Operating in function space.}

It has been shown (Krasnoselski [1], p. 128), that the generalized Borsuk theorem as stated above is also valid for completely continuous vectorfields in a Banach space. Here we make use of the fact, that certain periodic boundary value problems are equivalent to integxal equations of Hammerstein type. A further step would be to treat periodic boundary value problems, where the Hammerstein operator does not exist but a type of generalized Hammerstein operator introduced by MaWHIN ([2]). 
From system (1) we pass to the system

$$
\begin{aligned}
& \dot{x}=A x+f(x, y, t) \\
& \dot{y}=B y+g(x, y, t)
\end{aligned}
$$

where $A$ is a $p \times p$ matrix and $B$ a $(n-p) \times(n-p)$ matrix. We suppose that the linear homogeneous systems $\dot{x}=A x$ and $\dot{y}=B y$ have only the trivial $\omega$-periodic solution.

Let $B_{1}$ resp. $B_{2}$ be the Banach space of continuous $\omega$-periodic $p$-vector resp. $(n-p)$ vector functions with the norm

$$
\|x\|=\max _{\boldsymbol{0} \leqslant t \leqslant \omega}|x(t)|
$$

| | being the euclidean norm in $R^{p}$ resp. $R^{n-p}$. In the direct sum $B=B_{1}+B_{2}$ of the linear spaces $B_{1}$ and $B_{2}$ we introduce a norm in the following way:

$$
\text { for } x \in B_{1}, y \in B_{2} \quad \text { let } \quad\|x+y\|=\max \left(\|x\|_{B_{2}},\|y\|_{B_{2}}\right) \text {. }
$$

Then $B$ is a Banach space too.

It follows from our assumptions, that the linear differential operators $L=d / d t-A$ and $M=d / d t-B$, defined on $B_{1}$ resp. $B_{2}$ have an inverse. It is known that $L^{-1}$ and $M^{-1}$ are linear and bounded. Let

$$
\begin{aligned}
& F(x, y)=L^{-1}[f(x(t), y(t), t)] \\
& G(x, y)=M^{-1}[g(x(t), y(t), t)]
\end{aligned}
$$

$F$ and $G$ are completely continuous operators defined on $B$ with values in $B_{1}$ resp. $B_{2}$. The existence of $\omega$-periodic solutions of $(7)$ is equivalent to the existence of zeros of the completely continuous vector field

$$
\Phi(z)=\left\{\begin{array}{l}
x-F(x, y) \\
y-G(x, y)
\end{array}\right\} \quad z=\left(\begin{array}{l}
x \\
y
\end{array}\right)
$$

Lev the subspace $B_{1}$ play the role of $U$ in the Introdnction and let $P$ be the projection on $B_{1}$. We have $P \Phi(z)=x-F(x, y)$ and condition $D$ may be written:

$$
\left\{\begin{aligned}
x-F(x,-y) \\
-y-G(x,-y)
\end{aligned}\right\} \text { and } \quad\left\{\begin{array}{c}
x-F(x, y) \\
-y+G(x, y)
\end{array}\right\} \quad \begin{aligned}
& \text { have nowhere } \\
& \text { opposite direction }
\end{aligned}
$$

This means, that for no $\lambda<0$ the identities

$$
\begin{gathered}
x-F(x,-y)=\lambda(x-F(x, y)) \\
-y-G(x,-y)=\lambda(-y+G(x, y))
\end{gathered}
$$


Heimut Krolte: Periodic solutions of ordinary differential equations, ete. 243

hold simultaneously. If we put $\mu=1 /(1-\lambda), 1-\mu=(-\lambda /(1-\lambda))$, these identities become

$$
\begin{aligned}
& x=\mu F(x, y)+(1-\mu) F(x,-y) \\
& y=\mu G(x, y)-(1-\mu) G(x,-y) \quad 0<\mu<1 .
\end{aligned}
$$

Now we are able to prove

Proposition 2. - The system

$$
\begin{aligned}
& \dot{x}=A x+f(x, y, t) \\
& \dot{y}=g(x, y, t)
\end{aligned}
$$

with $A$ as in (7) and the further conditions

a) $\quad \lim _{|x|+|y| \rightarrow \infty} \frac{f(x, y, t)}{|x|+|y|}=0$

b) $y g(x, y, t)>0$ for $|y| \geqslant r$ and $|x| \leqslant \alpha|y|$ for some $\alpha>0$,

has at least onew-periodic solution.

REMARK. - The constant $\alpha$ ean be arbitrary small; but it seems that condition $b$ ) cannot be weakened to $y g(0, y, t)>0$ for $|y| \geqslant r$.

Proof. - We replace the second of (8) by

$$
\dot{y}=B y+g_{1}(x, y, t)
$$

with $g_{1}=g-B y$ and $B$ a matrix with the same property as in (7). Then $L^{-1}$ and $M^{-1}$ exist and $F(x, y)$ and $G(x, y)=M^{-1} g_{1}(x, y, t)$ are defined.

It follows from $a$ ) and the boundedness of $L^{-1}$, that for every $\delta>0$ there is a constant $R>0$ such that

$$
\|F(x, y)\| \leqslant \delta(\|x\|+\|y\|) \text { for } \quad\|x\|+\|y\|>R
$$

Now take $\delta$ so that $\delta<1$, and $\delta /(1-\delta) \leqslant \alpha, R_{0}$ so that $(9)$ is satisfied, and $R=\max \left(R_{0},(1+\alpha) r\right)$. Consider the sphere $S$, defined by $\|z\|=R$. On $S \cap B_{1}$ we have $y=0$ and $\|P(x, 0)\| \leqslant \delta\|x\|<\|x\|$ and therefore $P \Phi(z)=x-F(x, 0) \neq 0$.

Suppose the contrary of $D^{\prime \prime}$, that means that $E$ is fulfilled for some $z \in S$ and $\mu$, $0<\mu<1$. Then we have, since $\|x\|+\|y\| \geqslant R \geqslant R_{0}$

$$
\|x\| \leqslant \mu\|F(x, y)\|+(1-\mu)\|F(x,-y)\| \leqslant \delta(\|x\|+\|y\|)
$$


and therefore

$$
\|x\| \leqslant \frac{\delta}{1-\delta}\|y\| \leqslant \alpha\|y\|
$$

Furthermore it follows that $\|y\| \geqslant r$, for if $\|y\|<r$, then

$$
\|x\|+\|y\| \leqslant(\alpha+1)\|y\|<(\alpha+1) r \leqslant R .
$$

Let $|y(t)|$ attain its maximum in $t_{0}$, then we are sure that for $t=t_{0}|y| \geqslant r,|x| \leqslant \alpha|y|$ and $d / d t\left(y^{2}\right)=2 y \dot{y}=0$. But the second of $E$ is equivalent to the differential equation

$$
\dot{y}=\mu g(x, y, t)-(1-\mu) g(x,-y, t)
$$

which after multiplication with $y$ gives in virtue of $b$ )

$$
y \dot{y}>0 \text {. }
$$

So we have arrived at a contradiction. Condition $\left(\mathrm{D}^{\prime \prime}\right)$ is satisfied.

We know already that $\|x\|>\|F(x, 0)\|$ on $S \cap B_{1}$. Therefore the field $P \Phi(z)=$ $=x-F(x, 0)$ is homotopic to the identity and has rotation one. Now the generalized Borsuk theorem says that the rotation of $\Phi(z)$ on $S$ is odd and especially nonzero. Then the field $\Phi(z)$ must have a zero and system (8) a periodic solution.

It should be mentioned that proposition 1 can be proved very directly by use of the Leray-Schauder method. For this scope we add and subtract $A x$ resp., $B y$ to the first resp. second equation of (1), $A$ and $B$ being matrices as in (7), but in addition $A$ shall be positive definite and $B$ positive (negative) definite. So we can again introduce the completely continuous vector field $\Phi(z)$. We consider the family of vector fields

$$
\Phi(z, \lambda)=\left\{\begin{array}{l}
x-\lambda F(x, y) \\
y-\lambda G(x, y)
\end{array}\right\} \quad 0 \leqslant \lambda \leqslant 1
$$

Obviously $\Phi(z, 1)=\Phi(z), \Phi(z, 0)=z$. We show that the possible solutions of $\Phi(z, \lambda)=0,0 \leqslant \lambda<1$, that is

$$
\begin{array}{ll}
\dot{x}=A x+\lambda(f(x, y, t)-A x) & x(0)=x(\omega) \\
\dot{y}=B y+\lambda(g(x, y, t)-B y) & y(0)=y(\omega)
\end{array}
$$

have a bound which is independent of $\lambda$. Let $z=\left(\begin{array}{l}x \\ y\end{array}\right)$ be such a solution, and suppose at first $\|y\| \leqslant \alpha\|x\|$. If the maximum of $|x|$ is attained in $t_{0}$, then we have $|y| \leqslant \alpha|x|$ and $x \dot{x}=0$ for $t=t_{0}$. Multiplying the first of (10) by $x$ we get

$$
(1-\lambda) x A x+\lambda x f\left(x, y, t_{0}\right)=0
$$


HeLMut KNoLdE: Periodic solutions of ordinary differential equations, eto. 245

and, looking at $a$ ), we conclude $\|x\|=\left|x\left(t_{0}\right)\right|<r$ and $\|y\|<\alpha r$. If in the other case $\|y\|>\alpha\|x\|$, we have $y \dot{y}=0$ and $|y|>\alpha|x|$ for some $t_{1}$. Multiplying the second of (10) by $y$ we get

$$
(1-\lambda) y B y+\lambda y g\left(x, y, t_{1}\right)=0
$$

and from this and condition $b)$ follows $\|y\|=\left|y\left(t_{1}\right)\right|<r$ and $\|x\|<(1 / \alpha) r$.

Thus there is a sphere on which $\Phi(z, \lambda) \neq 0$ for $0 \leqslant \lambda<1$.

If this holds for $\lambda=1$ too, then $\Phi(z)$ is homotopic to the identity and has rotation one. But this implies the existence of at least one $\omega$-periodic solution of (1). If $\Phi(z, 1)=0$ for some $z$, of course this is an $w$-periodic solution of (1).

\section{REFERENCES}

[1] M. A. Krasnoselski, Topological Methods in the Theory of Nonlinear Integral Equations, English Translation: Pergamon Press, 1964.

[2] J. Mawhin, A Generalization of Hammerstein Operators and Applications, Institut de math. pure et appl. de l'Univ. Cath. Louvain, Rapport no. 42, novembre 1971.

[3] V. A. Pliss, Nonlocal Problems in the Theory of Oscillations, English translation, New York, 1966.

[4] R. REISSIG, Anwendungen von Fixpunktsätzen auf das Problem der periodischen Lösungen bei nicht-autonomen Systemen, Deutsche Luft. und Raumfahrt, Forschungsbericht 69-53, 1969. 